\title{
Design of Double Environment Dynamical Simulation System for Fuze
}

\author{
Bin Zhang a , Qiang Xiao, Shiyin Huang, Han Wei, Manyi Ding \\ No. 63981 Unit of PLA, Wuhan, 430311, China \\ a Xiaosongqiang8@163.com
}

\begin{abstract}
Dynamic simulation is an effective way to test the fuze performance. The simulation realization of fuze environmental is the premise and foundation of fuze performance test. Simulation fidelity directly determines whether the fuze quality data is scientific and effective. In order to accurately and dynamically simulate double environment force field of centrifugal force and the target effect with the fuze at the time of ammunition launch, in the current column installed fuze as the test object, double environment simulation dynamical system for fuze is developed based on PLC. Firstly, the basic idea PLC control system is introduced briefly. Then the overall design of the system is completed, and framework of the system is given based on it. Finally, the system function is researched and the working mechanism is determined, system automatic control and information exchange is realized. It is of some reference value for the fuze study method and system development.
\end{abstract}

Keywords: Programmable Logic controller (PLC); Fuze; double environment; Simulation System.

\section{Introduction}

As the "brain" and the core of ammunition, the fuze is the terminal main body to damage target. The performance of the fuze has a serious impact on operational efficiency of ammunition. It has been a hot topic to study how to detect the comprehensive performance of the fuze scientifically and effectively.

According to the problem that subentry and static test method cannot test fuze performance comprehensively and accurately. At present, related research of centrifuge and target effect double environment force simulation is rarely involved, the design and research of the simulation system is rarer. Usually this chronicle of the trial is the optimal technique for Fuze performance test means, but by long time-consuming, confined space, high cost and poor repeatability, and through the effective simulation of fuze double environment completing comprehensive test has become is the most effective and economical technology by far in military field with low cost, safety and security, flexible control, small weather effects and any field operations unique advantages.

From the urgent need of the comprehensive performance test of Fuze in the paper, Based on actual situation, according to the actual and structural characteristics of the unit ammunition fuze, fuze double environment simulation system of fuze is develop based on PLC control system platform, the composition of system is introduced briefly, and the function of the subsystem is allocated reasonably. On the basis of this, the operating mechanism of the simulation system is designed.

\section{PLC overview}

With the rapid development of control technology, as one of the advanced industrial computer control system, the PLC has been widely used in the military field, which brings good technical results and economic benefits to the military departments. Therefore, it is a practical and feasible method to study dynamical double environment force simulation system for Fuze [1].

PLC is oriented to the idea of digital logic operations to establish the control system, and the control system is considered as a "brain" [2], each equipment of the control system is a module of the system, each module can relatively independent designed and develop to the maximum degree by itself in the field of advanced technology, and each module is provided with more network communication service interface layer for the operation of the PLC system, operation of the system is ensure efficient and accurate, and the three aspects of system function realization of the control algorithm program, operation management of equipment and underlying information interactive 
transmission is effectively separated, the operation, expansion of follow-up system can is improved The logical structure of the PLC control system is shown in Fig 1.So by using the standard framework of PLC, the simulation can be established.

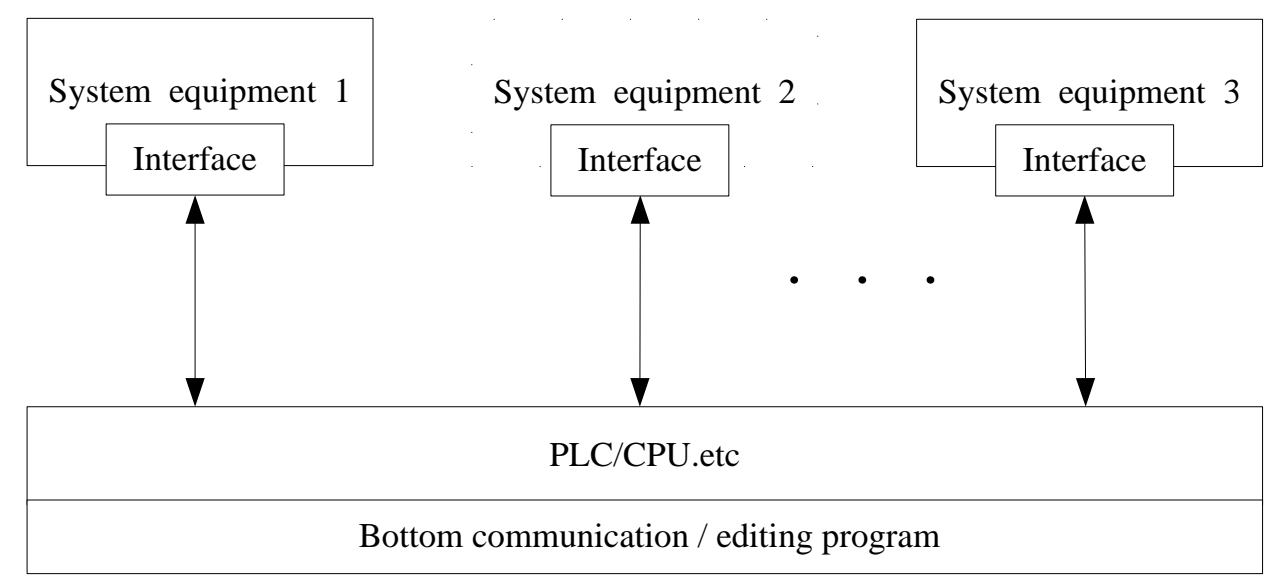

Fig. 1 The logical structure of the PLC control system

\section{Simulation System Construction}

In the support of PLC, dynamical double environment simulation system for Fuze designed and develop, from the demand and function of the detection system, the overall structure of system is divided into pre dump energy transmission system, target effect system, PLC control system, man-machine interface etc four parts. By using modular and integrated design method, modularization design, pre dump energy system, target points system is comprehensive integrated as the double environment simulation platform, PLC control system, man-machine interface is set as a guide control platform.

\section{Simulation System Design}

\subsection{Pre Dump energy Transmission System}

The system is an important part of the dynamic simulation test for fuze which is used to simulate the fuze centrifugal environment [3]. It is mainly made of pre dump energy device for providing centrifugal kinetic energy including frequency converter for speed control and AC motor for rotation drive, tachometer for getting rotate speed information, and centrifugal device for fixing and driving the fuze. Its structure schematic diagram is shown in Fig 2.

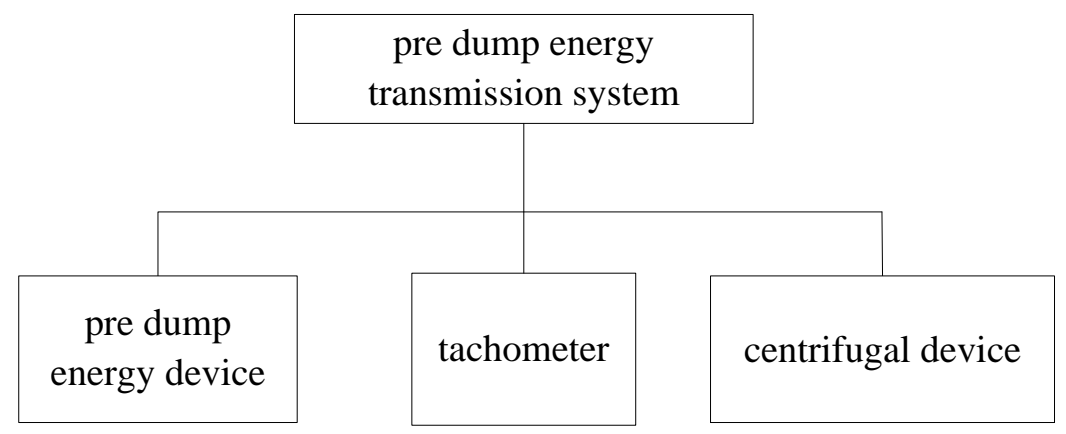

Fig. 2 The structure schematic diagram of pre dump energy transmission system

The closed-loop dynamic negative feedback control mode is adopted to modify the rotation error automatically, and then the rotation speed and high precision control are realized. According to the working principle of the rotary drive system, its function structure diagram is shown in fig 3 . 


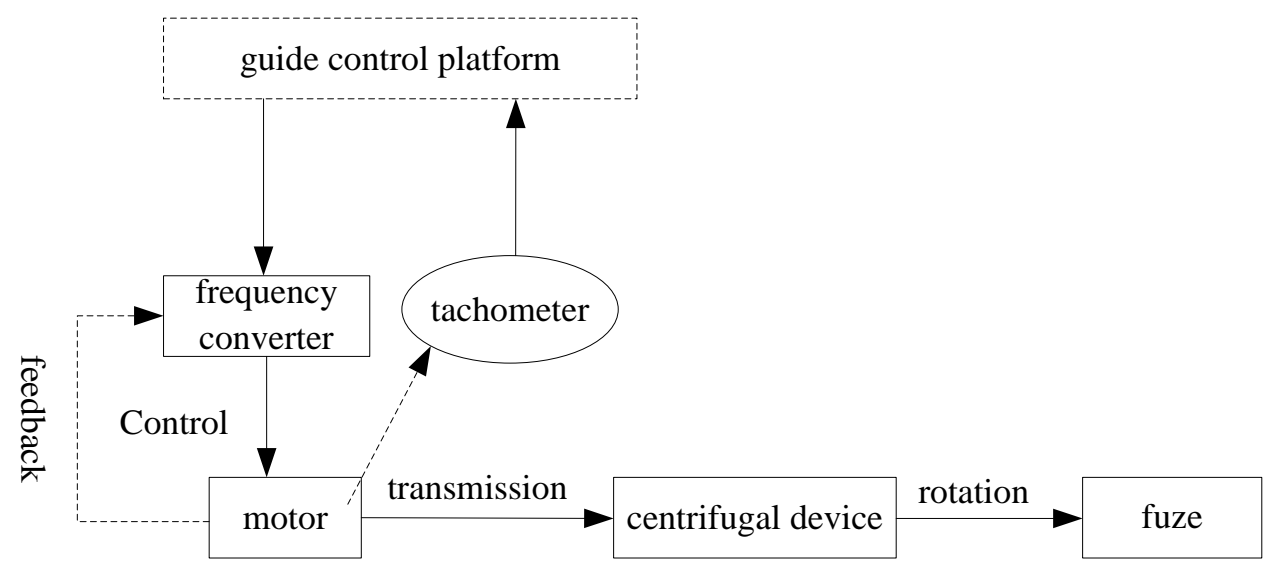

Fig. 3 function structure diagram

\subsection{Target Effect System}

The main task of the target function system is used to simulating the environment of the target effect, and to finishing the test of the fire resistance performance of the fuze terminal trajectory [4]. In the design, the system is mainly made of target shock device for simulating "target" impact fuze, pressure storage energy device for improving target impactor velocity, target guiding device which can be used to controlling the effect of target direction of movement and electromagnetic unlocking device for controlling target impactor device attack time precisely, the system structure schematic diagram as shown in Fig. 4.

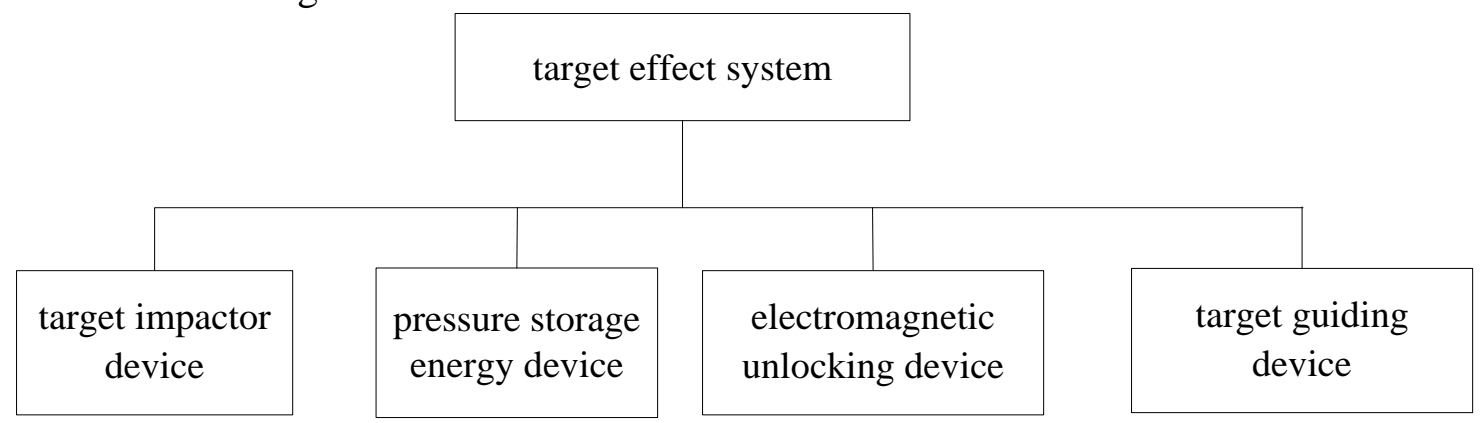

Fig. 4 The structure schematic diagram of the target effect system

The effective control of the target effect system is the key to the fire test of the fuze. According to the demand of fire energy, in order to realize reliable fire resistance performance test, the system function structure diagram is given in Fig. 5.

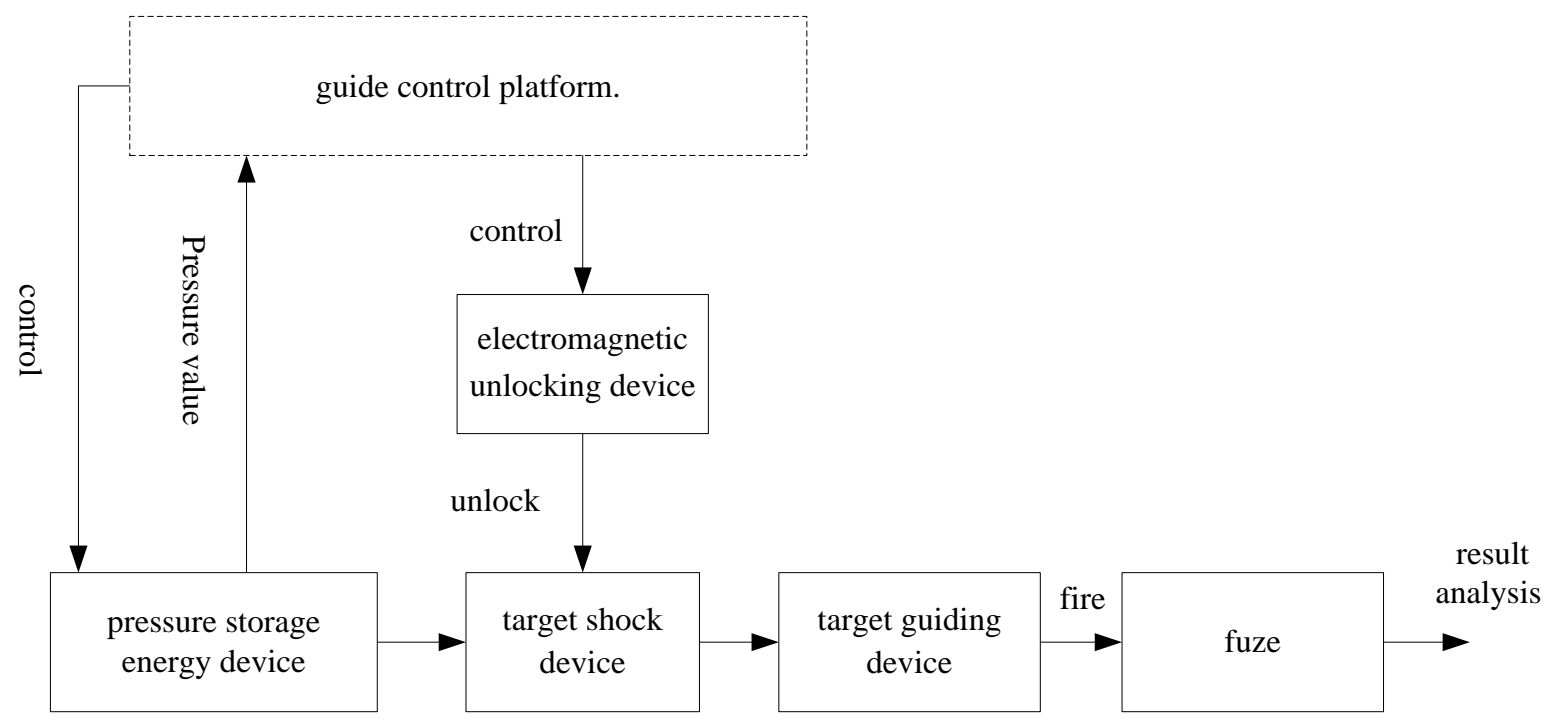

Fig. 5 function structure diagram 


\subsection{Man-machine Interface}

It is interaction information window between human-computer that is mainly used to setting system initial state parameters, different types fuze test parameters, selecting operation mode, monitoring system running state and perform the system start, end, emergency stop and operation control in test process.

\subsection{PLC Control System}

It is the core component of the whole system. The measurement and control performance of the system determines the automatic and intelligent level of the fuze performance test. The system is mainly used to completing the automatic control function of the double environment simulation platform [5]. The precision control of the pre dump energy system and the target effect system is realized to ensure the simulation system peration efficient.

\section{Conclusion}

Double environment simulation system of fuze is develop and designed by the PLC, through reasonable division of system and distribution function, and the operation mechanism of the system is analyzed in depth, which provides an effective and feasible method for double environment simulation system for fuze developed. Because this paper focuses on double environment simulation system design process, the specific system is only introduced briefly, but the design theory and method in the study is general. It has a certain reference military value to promote the rapid development of ammunition fuze test system. In the future research, the PLC measurement and control system specific programming development e $\mathrm{n}$ study will be carry out.

\section{References}

[1]. Liu Meng. Based on PLC and WinCC Fuze Automatic Assembly Aachine Control System Design [D]. Sichuan ordnance journal, Vol. 36(2015) No. 3, p.31-34.

[2]. .LV Pin. Application of PLC and Touch Panel Control System [J]. Automation Instrument. Vol. 31 (2010) No. 8, p.45-47 Vol. 31(2010) No. 8, p.45-47.

[3]. Yang Yun-sheng, Feng Shao-wei. A Synthesis Method for Naval Gun Hydraulic Components Detection [J]. Rocket Launch and Control Journal. Vol. 37 (2016) No. 2, p.75-78.

[4]. Ma Shao-jie. Fuze test technology [M]. Beijing: National Defense Industry Press, 2010, p.45-64.

[5]. Zhang Yang. Design and Implementation of Intelligent Material Installation System based on PLC and Configuration Software [D]. Master's Degree Thesis of Jilin University, 2012, p.14-26. 